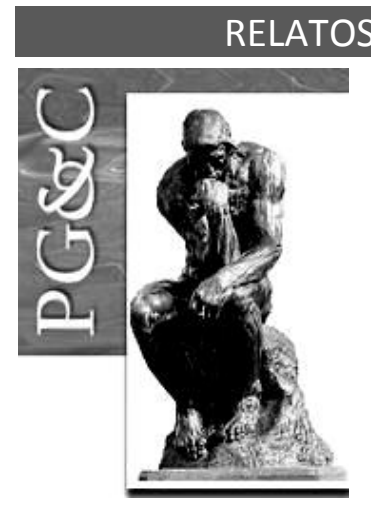

\title{
A GESTÃO DO CONHECIMENTO HOLÍSTICA: ANÁLISE DE ADERÊNCIA DO MODELO DE TEIXEIRA FILHO (2000)
}

\author{
Fábio Corrêa \\ Doutor em Sistemas de Informação e Gestão do Conhecimento \\ pela Universidade FUMEC, Brasil. \\ E-mail: fabiocontact@gmail.com \\ Fabrício Ziviani \\ Doutor em Ciência da Informação pela UFMG, Brasil. \\ Professor da Universidade FUMEC, Brasil. \\ E-mail: fazist@hotmail.com \\ Henrique Cordeiro Martins \\ Doutor em Administração pela UFMG, Brasil. \\ Professor da Universidade FUMEC, Brasil. \\ E-mail: henrique.martins@hotmail.com
}

\begin{abstract}
Resumo
O paradigma holístico é uma perspectiva apontada para que a Gestão do Conhecimento aborde o conhecimento organizacional de forma abrangente e unificadora. Considerando ser essa uma perspectiva necessária para o desenvolvimento de modelos de Gestão do Conhecimento, esse estudo objetiva analisar a aderência do modelo de Teixeira Filho (2000) em relação ao que se denomina por Gestão do Conhecimento holística. Por meio de procedimentos metodológicos mistos e fazendo uso da Análise de Conteúdo o referido modelo foi analisado. Por resultado, o modelo de Teixeira Filho (2000) adere parcialmente a Gestão do Conhecimento holística. Isto permite concluir que há necessidade de desenvolvimento de modelos mais abrangentes, orientados a todas as dimensões da Gestão do Conhecimento e suas relações de forma a atingir o hólus dessa temática no âmbito organizacional. Esta pesquisa contribui com a academia por apresentar um delineamento da Gestão do Conhecimento ao paradigma holístico e por prover a análise integra de um modelo quanto a sua aderência a essa forma de gestão. Sugestão de desenvolvimento de um modelo holístico para a Gestão do Conhecimento e de análises de aderência de outros modelos são propostas de pesquisas futuras.
\end{abstract}

Palavras-chave: Gestão do Conhecimento. Paradigma holístico. Gestão do Conhecimento holística. Teixeira Filho. Análise de Conteúdo.

\section{THE HOLISTIC KNOWLEDGE MANAGEMENT: ADHERENCE ANALYSIS OF THE TEIXEIRA FILHO (2000) MODEL}

\begin{abstract}
The holistic paradigm is a pointed perspective for Knowledge Management to approach organizational knowledge in a comprehensive and unifying way. Considering that this is a necessary perspective for the development of Knowledge Management models, this study aimed to analyze the adherence of the model of Teixeira Filho (2000) in relation to what is called by Holistic Knowledge Management. By means of mixed methodological procedures and making use of Content Analysis, said model was analyzed. As a result, the model of Teixeira Filho (2000) partially adheres to holistic Knowledge Management. This leads to the conclusion that there is a need to develop more comprehensive models oriented to all dimensions of Knowledge Management and their relationships in order to reach the holos of this subject in the organizational scope. This research contributes to the academy by presenting a delineation of Knowledge Management to the holistic paradigm and by providing the integrated analysis of a model
\end{abstract}

Perspectivas em Gestão \& Conhecimento, João Pessoa, v. 8, número especial, p. 167-182, out. 2018. DOI: http://dx.doi.org/10.21714/2236-417X2018v8nep167

http://periodicos.ufpb.br/ojs2/index.php/pgc. ISSN: 2236-417X. Publicação sob Licença (cc) EY-NC-ND 
regarding its adherence to this form of management. Suggestion for developing a holistic model for Knowledge Management and for adherence analysis of other models are proposed for future research.

Keywords: Knowledge management. Holistic paradigm. Holistic Knowledge Management. Teixeira Filho. Content analysis.

\section{INTRODUÇÃO}

Até o presente momento a forma de se fazer ciência é alicerçada no método reducionista dos matemáticos René Descartes (1596-1650) e Isaac Newton (1642-1727). O método reducionista, também denominado newtoniano-cartesiano em homenagem aos seus propositores, se propõe a dividir um fenômeno em partes menores e estudá-las isoladamente para posterior compreensão do fenômeno em sua totalidade (VERGARA, 1993; PEREIRA, 2002; FERREIRA, 2009; CREMA, 2015).

Essa forma de se abordar um fenômeno cientificamente é a base das ciências atuais, como os algoritmos da Ciência da Computação (CORMEN et al. 2002; ZIVIANI, 2014). Esses algoritmos subdividem um problema em módulos e os resolvem de modo isolado, levando a solução do problema maior por meio da junção das menores partes solvidas. A educação também promove esse método, uma vez que as "as escolas repartiram o conhecimento em áreas, as áreas em cursos, os cursos em disciplinas, as disciplinas em especificidades" (BEHRENS, 1999, p. 384), tornando as universidades verdadeiras torres de Babel (WEIL, 2001).

No campo organizacional a Administração Científica de Taylor e Ford (ANDRADE; AMBONI, 2017) reduziu o homem a uma engrenagem de uma máquina industrial, simplificando a complexidade humana por uma perspectiva mecânica que desconsidera suas emoções e pensamentos (MACIEL; SILVA, 2008). Em outros termos

Taylor manda executar movimentos sob rígido controle e Ford manda adaptar movimentos tão comodamente quanto possível ao ritmo de produção, às aptidões e à vontade. Taylor se preocupou mais com o estudo do tempo perdido pelo homem e pela máquina; Ford procurou suprimir o tempo-perdido pela matéria-prima, com o trabalho contínuo (SILVA, 2013, p. 122).

Essa simplificação chegou as estruturas organizacionais, que se subdividem uma empresa em organogramas, áreas, setores, cargos e funções. Por conseguinte, o que se tem é um processo simplificador, reducionista e fragmentador que trata os problemas em porções simplistas que, em verdade, diminuem a complexidade do todo e torna as análises e soluções não aderentes a realidade. Para Weil (1991) e Coimbra (1996) o reflexo desse modo de fazer ciência é responsável pelas especializações e pela visão mecânica do mundo.

Embora esse método seja pontuado como lesivo para a humanidade (WEIL, 1991; COIMBRA, 1996), todos os avanços científicos desfrutados até a atualidade são decorrentes dessa forma de se fazer ciência. O lançamento do Sputnik, em 1957 (CREMA, 2015), a chegada do homem à lua (PEREIRA, 2002) até a evolução do pensamento humano (FLACH; BEHRENS, 2008) são resultados desse método. Todavia, o que se pondera é a necessidade de abordar os problemas de uma forma diferente da reducionista, sendo essa forma denominada holística.

Holism and evolution foi uma publicação do general sul-africano e filósofo lan Christian Smuts, datada de 1926 (WEIL, 1991; CREMA, 1991; VERGARA, 1993; PEREIRA, 2002, MACIEL; SILVA, 2008; CREMA, 2015), que impulsionou a perspectiva de um novo modo de se fazer ciência. O holísmo também visa promover a compreensão de um fenômeno considerando suas partes, no entanto diverge do reducionismo por não analisar essas partes isoladas, mas sim

Perspectivas em Gestão \& Conhecimento, João Pessoa, v. 8, número especial, p. 167-182, out. 2018. 
dentro do contexto para que as conexões entre as partes sejam mantidas, pois essas conexões também constituem o todo (WEIL, 1991).

Dessa forma a visão holística assume que o fenômeno pode ser compreendido em sua integralidade, já que considera as relações entre as partes. Isso a distingue da visão reducionista que desconsidera essas relações uma vez que analisa os elementos de forma disjunta do todo (CAPRA, 2000). É sobre esse delineamento científico que a Gestão do Conhecimento $(\mathrm{GC})$ holística se estabelece.

A GC atua nas organizações que, como posto anteriormente, são seccionadas em estruturas. Além de atuar em um contexto fragmentado a GC também é seccionada em partes, como pessoas, tecnologia e processos, secção essa abordada no modelo de GC de Angeloni (2002) e assumida com outras variância no modelo de Terra (2005). A visão holística é demarcada para a GC como uma forma de compreender o todo considerando suas partes em conjunto, juntamente com suas propriedades emergentes das inter-relações entre as partes (PEREIRA, 2002).

Essa perspectiva do holísmo para com a GC foi pontuada nos estudos de Holsapple e Joshi (1999), Rubenstein-Montano et al. (2001) e Heisig (2009) que analisaram 196 modelos de GC e acenaram a necessidade de um modelo que seja unificador, abrangente, holístico. Conforme Heisig (2009), abordagens que consideram apenas um elemento (parte) são insuficientes para a GC, pois "o objetivo da GC é implementar uma abordagem holística" (HEISIG, 2009, p. 16, tradução dos autores).

Todavia, emerge os seguintes questionamentos: quais as partes que compõem uma GC holística? A GC tem caminhado para abordagens que considera a visão holística? Essas duas perguntas são problemáticas tratadas nesse estudo como necessárias para atingimento do objetivo dessa pesquisa que visa: analisar a aderência do modelo de Teixeira Filho (2000) a GC holística. Uma vez que para entender o todo é necessário conhecer as partes, pois a visão holística assume a secção (VERGARA, 1993; PEREIRA, 2002), o primeiro questionamento visa identificar os elementos que compõem a GC holística.

Feito isso, a segunda questão tende a ser investigada. Sabendo-se das partes que constituem o todo da GC, busca-se investigar se algum modelo de GC analisou todos os elementos vistos como essenciais para uma abordagem holística orientada ao conhecimento nas organizações. Mediante ao elevado quantitativo de modelos -196 analisados somente por três estudos supracitados -, esse estudo se propõem a analisar o modelo de Teixeira Filho (2000) em relação a GC holística. Assim, acredita-se que essa pesquisa inicia uma caminhada para compreender a abrangência e convergência de modelos de GC ao que se denomina por uma abordagem holística voltada para o conhecimento.

Destarte, para atingimento de seu intento esse estudo se subdivide em seções. Além dessa introdução, a seção seguinte (seção 2) delineia as partes (dimensões) que conformam a GC holística. Em sequência (seção 3) os procedimentos metodológicos são expostos para determinar os métodos empregados para análise do modelo de Teixeira Filho (2000) quanto a aderência a GC holística. A seguir (seção 4) a análise do modelo é realizada seguindo os métodos explanados (subseção 4.1) e os resultados são expostos (subseção 4.2). Por fim, as considerações finais são apresentadas (seção 5) e explicitadas as referências utilizadas ao longo dessa pesquisa.

\section{DIMENSÕES DA GESTÃO DO CONHECIMENTO HOLÍSTICA}

A seção anterior delineou a visão reducionista e a holística, ajustando essa segunda a GC. Uma vez que o holísmo assume que as partes devem ser analisadas junto ao seu todo (PEREIRA, 2002), sendo "o objetivo da GC é implementar uma abordagem holística" (HEISIG,

Perspectivas em Gestão \& Conhecimento, João Pessoa, v. 8, número especial, p. 167-182, out. 2018. 
2009, p. 16, tradução dos autores), se torna necessário identificar e compreender quais são essas partes.

Rockart (1979) definiu Fatores Críticos de Sucesso (FCS) como áreas das quais, se os resultados forem satisfatórios, garantirão o desempenho organizacional bem-sucedido. Para a GC esses fatores são vistos como vitais para que investidas em âmbito organizacional contemplem áreas que são imperativas para o sucesso desta forma de gestão (KUMAR; SINGH; HALEEM, 2015). Portanto, os FCS constituem as dimensões, ou partes, da GC holística, sendo necessária atenção a essas em investidas orientadas ao conhecimento.

Mediante a essa compreensão o total de 18 estudos - com recorte temporal de 1997 a 2015 - acerca dos FCS da GC foram analisados e consolidados, gerando 13 FCS (partes) as quais a GC deve se orientar. Esses estudos são expostos de forma a fundamentar tais fatores, que são Estratégia (1), Liderança e suporte da alta administração (2), Equipe de gestão do conhecimento (3), Recursos (financeiro, humano, material e tempo) (4), Processos e atividades (5), Gestão de recursos humanos (6), Treinamento e educação (7), Motivação (8), Trabalho em equipe (9), Cultura (10), Tecnologia da informação (11), Mensuração (12) e Projeto Piloto (13).

A Estratégia (1) condiz com a determinação de objetivos e planos para seu atingimento (ALTAHER, 2010). A GC se ajusta a estratégia organizacional pelo alinhamento e clarificação. $O$ alinhamento resulta em convergir o objetivo da GC em relação a estratégia organizacional (AKHAVAN; JAFARI; FATHIAN, 2006). Assim, o programa de GC deve apoiar os objetivos estratégicos da empresa por meio de proposta de valor (AL-MABROUK, 2006), ainda que não seja possível que esse valor seja calculado diretamente (SKYRME; AMIDON, 1997). A clarificação remete a comunicação dessa estratégia a todos os membros da organização (WONG, 2005; LIN, LIN, 2006; GAl; XU, 2009; ABBASZADEH; EBRAHIMI; FOTOUHI, 2010).

A Liderança e suporte da alta administração (2) consiste no apoio da alta administração e de seus líderes no aspecto financeiro (DAVENPORT; DE LONG; BEERS, 1998; GAI; XU, 2009; NAGHAVI; DASTAVIZ; NEZAKATI, 2013), moral (AL-MABROUK, 2006), de tempo (ZIEBA; ZIEBA, 2014); ARIF; SHALHOUB, 2014), direcionamento de conhecimentos relevantes (DAVENPORT; DE LONG; BEERS, 1998), bem como contribuir para homogeneizar a mensagem da GC na organização (AL-MABROUK, 2006).

A Equipe de gestão do conhecimento (3) resulta em definir papéis e responsabilidades voltadas para projetos de conhecimento (DAVENPORT; DE LONG; BEERS, 1998; WONG; ASPINWALL, 2005; VALMOHAMMADI, 2010; ARIF E SHALHOUB, 2014), definir um chefe de conhecimento (WONG; 2005; GAI; XU; 2009; SEDIGHI; ZAND, 2012) e definir uma equipe de profissionais orientados a promoção da GC na organização (DAVENPORT; DE LONG; BEERS, 1998; WONG, 2005; WONG; ASPINWALL, 2005; AL-MABROUK, 2006; LIN; LIN, 2006; GAI; XU, 2009; VALMOHAMMADI, 2010; ARIF; SHALHOUB, 2014; SEDIGHI; ZAND, 2012).

Os Recursos (financeiro, humano, material e tempo) (4) remetem as necessidades de insumos para a promoção dos projetos de GC, como financeiro (AL-MABROUK, 2006; ABBASZADEH; EBRAHIMI; FOTOUHI, 2010), humano (WONG, 2005), material (SEDIGHI; ZAND, 2012; KUMAR; SINGH; HALEEM, 2015) e de tempo (AL-MABROUK, 2006). Esses são necessários em qualquer iniciativa organizacional e, da mesma forma, são críticos para o desenvolvimento de projetos de conhecimento.

Os Processos e atividades (5) são o centro da GC por atuarem sobre o manuseio do conhecimento e elucidam o que deve ser feito com o conhecimento (WONG, 2005; ABBASZADEH; EBRAHIMI; FOTOUHI, 2010). Assim se deve estabelecer os processos de GC da organização (VALMOHAMMADI, 2010; SEDIGHI; ZAND, 2012) e integrá-los ao fluxo de trabalho para que sejam desempenhados no cotidiano da empresa (GAl; XU (2009).

A Gestão de recursos humanos (6) é a área central que atua com as pessoas, essas detentoras do conhecimento (VALMOHAMMADI, 2010; SEDIGHI; ZAND, 2012). Promover a contratação de pessoas de acordo com as lacunas de conhecimento (WONG, 2005; WONG;

Perspectivas em Gestão \& Conhecimento, João Pessoa, v. 8, número especial, p. 167-182, out. 2018. 
ASPINWALL, 2005), o desenvolvimento desses indivíduos (WONG, 2005; KUMAR; SINGH; HALEEM, 2015), bem como a retenção dos mesmos (WONG, 2005) por meio de políticas de carreiras, por exemplo, são atividades que apoiam o gerenciamento do conhecimento.

O Treinamento e educação (7) remete ao intento de prover uma linguagem clara quanto aos termos da GC. Homogeneizar conceitos, vocábulo e o intento da GC (ALMABROUK, 2006; AKHAVAN; JAFARI; FATHIAN, 2006), o propósito e os papéis da equipe de GC (DAVENPORT; DE LONG; BEERS, 1998; WONG; ASPINWALL, 2005), habilidades para uso das ferramentas de conhecimento (ABBASZADEH; EBRAHIMI; FOTOUHI, 2010; ANGGIA, 2013), flexibilidade na aceitação de erros (SKYRME; AMIDON, 1997; ARIF; SHALHOUB, 2014) e educar as pessoas para os quesitos anteriores (KUMAR; SINGH; HALEEM, 2015) são pontos a serem considerados por esse fator (dimensão).

A Motivação (8) visa o desenvolvimento de aspectos motivacionais para que as tratativas do conhecimento sejam desempenhadas, como plano de carreira e avaliação dos funcionários (WONG, 2005; SEDIGHI, ZAND; 2012; ZIEBA; ZIEBA, 2014), sistema de motivação de recompensas, gratificação e, ou, reconhecimento (WONG; ASPINWALL, 2005; VALMOHAMMADI, 2010; ZIEBA; ZIEBA, 2014; ARIF; SHALHOUB, 2014) e sinalização de práticas motivacionais a gestão de recursos humanos para a criação, trabalho em equipe e comportamento colaborativo (AL-MABROUK, 2006; LIN; LIN, 2006; GAI; XU, 2009; VALMOHAMMADI, 2010).

O Trabalho em equipe (9) remete ao fomento para que trabalho em equipe, com vistas ao compartilhamento e criação de novos conhecimentos, seja realizado (AKHAVAN; JAFARI; FATHIAN, 2006; WAI; HONG; DIN, 2011; SEDIGHI; ZAND; 2012). A Cultura (10) condiz com o estabelecimento de uma cultura tolerante a erros e que reconheça potencial de aprendizado por meio desses (WONG, 2005).

A Tecnologia da informação (11) suscita o provimento de acesso ao conhecimento explícito por meio de mídias digitais, intranet, extranet, dentre outros (DAVENPORT; DE LONG; BEERS, 1998; ARIF; SHALHOUB, 2014) e conexão dos indivíduos por meio de videoconferências e outros recursos tecnológicos em prol do compartilhamento do conhecimento tácito (SKYRME; AMIDON, 1997; WONG; ASPINWALL, 2005; SEDIGHI; ZAND, 2012; KUMAR; SINGH; HALEEM, 2015).

A Mensuração (12) remete a vertente de capital intelectual. Busca o estabelecimento de indicadores para medição da GC (AL-MABROUK, 2006; GAI; XU, 2009; SEDIGHI; ZAND, 2012), estabelecimento de formas de medição (DAVENPORT; DE LONG; BEERS, 1998), diagnóstico e avaliação da GC (AKHAVAN; JAFARI; FATHIAN, 2006; ARIF; SHALHOUB, 2014) e comunicação dos resultados a alta administração para apoio contínuo (WONG, 2005; ALMABROUK, 2006).

Por fim, o Projeto Piloto (13) busca o desenvolvimento de projetos em menor âmbito organizacional para aprendizagem antes de uma implementação em maior amplitude (SKYRME; AMINDON, 1997; AKHAVAN; JAFARI; FATHIAN, 2006). Todos esses FCS são as partes, ou dimensões, das quais a GC holística deve se atentar para o provimento do gerenciamento do conhecimento. Mediante ao estabelecimento dessas os procedimentos metodológicos para análise de aderência do modelo de Teixeira Filho (2000) a GC holística são delineados a seguir.

\section{PROCEDIMENTOS METODOLÓGICOS}

Segundo Gil (2002) uma pesquisa deve ser tipificada por sua natureza e delineamento teórico adotado para sua condução. Sob essa determinação, essa pesquisa é classificada quanto a natureza, abordagem, técnica de pesquisa e método de análise.

Gil (2002) revela que o objetivo de uma pesquisa delineia sua natureza. Assim, mediante ao intento de analisar a aderência do modelo de Teixeira Filho (2000) a GC holística,

Perspectivas em Gestão \& Conhecimento, João Pessoa, v. 8, número especial, p. 167-182, out. 2018. 
esse estudo é classificado como do tipo descritivo. Isso porque prima pela exatidão na descrição dos fenômenos (GIL, 2002) que, nesse estudo, se revela pela descrição da presença, ou ausência, dos fatores da GC holística no modelo analisado.

Quanto a abordagem, essa pesquisa é classificada como mutimétodo (CRESWELL, 2007), ou seja, qualitativa e quantitativa. Conforme Minayo (1998) a abordagem qualitativa permite captar significações acerca do fenômeno estudado, mas segundo Kerlinger (1979), essa admite interferências devido a subjetividade do observador. Assim, durante o processo qualitativo de análise do referido modelo de GC é buscado identificar os significados da obra de forma a posicioná-los mediante as dimensões da GC holística.

Por ser um processo de leitura e interpretação, admite-se, então a subjetividade. No entanto, a natureza descritiva e as citações diretas são utilizadas como alicerces do processo racional que, segundo Flick (2004) é necessário para manutenibilidade das significações apreendidas ao longo da análise.

A abordagem quantitativa se fundamenta na quantificação, na qual as análises valemse do uso de técnicas matemáticas (MINAYO, 1998; GIL, 2002). Mediante a identificação das dimensões na obra analisada, intento da abordagem qualitativa, os resultados também serão expostos quantitativamente, permitindo que se compreenda o grau percentílico de atendimento do modelo em questão ao que se denomina por GC holística.

A técnica de pesquisa condiz com os meios para se atingir o ambicionado, podendo fazer uso de documentação indireta, direta, direta intensiva e direta extensiva (MARCONI; LAKATOS, 2003). A documentação indireta abriga a técnica de pesquisa bibliográfica (MARCONI; LAKATOS, 2003), que faz uso de faz uso de artigos científicos, livros, dissertações, teses, dentre outros meios e mídias (BARROS; LEHFELD, 2007).

Nessa pesquisa é analisada, especificamente, a obra (livro) de Teixeira Filho (2000) que apresenta o modelo de GC do autor. Esse escolha é pautada no fato de que esse estudo influenciou modelos de GC, como os de Costa (2005), Hanashiro (2005) e Cajueiro (2008) e ainda é citada em estudos atuais (OLIVEIRA et al., 2012; MELO; MEDEIROS, 2017; ALVES, 2017), evidenciando sua relevância para a GC e justificando sua seleção para esse estudo.

Como método de análise essa pesquisa faz uso da Análise de Conteúdo que tem por insumo textos escritos, passíveis de serem manipulados para fornecer respostas ao objetivo de pesquisa (GASKELL; BAUER, 2002). Segundo Bardin (1977) esse método é

Um conjunto de técnicas de análise das comunicações visando obter, por procedimentos, sistemáticos e objectivos de descrição do conteúdo das mensagens, indicadores (quantitativos ou não) que permitam a inferência de conhecimentos relativos às condições de produção/recepção (variáveis inferidas) destas mensagens (BARDIN, 1977, p. 42, itálico original).

Por meio da análise do conteúdo da obra de Teixeira Filho (2000) as dimensões da GC holística são identificadas de forma a permitir posicionar sua aderência a essa temática. Todavia, para aplicar esse método é necessário estabelecer as unidades de registro, compreendidas como os seguimentos a serem extraídos do texto original para posterior processamento (BARDIN, 1977).

As unidades de registro refletem as dimensões da GC, assinaladas na seção anterior dessa pesquisa, sendo 13, a saber: Estratégia, Liderança e suporte da alta administração, Equipe de gestão do conhecimento, Recursos (financeiro, humano, material e tempo), Processos e atividades, Gestão de recursos humanos, Treinamento e educação, Motivação, Trabalho em equipe, Cultura, Tecnologia da informação, Mensuração e Projeto Piloto.

Em suma, a referida obra será analisada qualitativamente pela Análise de Conteúdo de forma a identificar as dimensões da GC holística e, posteriormente, quantitativamente para

Perspectivas em Gestão \& Conhecimento, João Pessoa, v. 8, número especial, p. 167-182, out. 2018. 
posicionar essa obra percentilicamente quanto a sua adesão ao holísmo almejado para a GC. Visando maior rigor científico as citações diretas serão empregadas de forma a permitir a manutenibilidade das dimensões apreendidas e exame das mesmas pelo leitor, bem como para evidenciar o percurso cognitivo que levou a promover interpretações acerca das dimensões identificadas na obra.

\section{APRESENTAÇÃO DA ANÁLISE E RESULTADOS}

Essa seção se subdivide em duas subseções. A primeira (subseção 4.1) apresenta a Análise de Conteúdo realizada sobre o modelo de Teixeira Filho (2000). Nessa análise o rigor metodológico é empregado de forma a evidenciar todo o percurso cognitivo que orienta a compreensão de presença, ou ausência, de uma dimensão no referido modelo. As citações diretas são costumeiramente utilizadas, visando permitir o regresso do leitor aos trechos da obra para melhor clareamento das inferências realizadas.

A segunda parte (subseção 4.2) é condicionada aos achados da subseção anterior. Nessa todas as dimensões são assinaladas quanto a sua presença no modelo e representadas de forma a permitir compreender a aderência do modelo as dimensões da GC holística. Ademais, também é promovida uma quantificação percentílica que permite compreender a aderência do modelo ao que se denomina por holísmo na GC.

\subsection{Análise do modelo de Teixeira Filho (2000)}

Teixeira Filho (2000) defende que a GC "pode ser vista como uma coleção de processos que governa a criação, disseminação e utilização do conhecimento para atingir plenamente os objetivos da organização" (TEIXEIRA FILHO, 2000, p. 22, 97, 103) e afirma que a GC não é para todos por que demanda fatores como organização, visão sistêmica, tecnologia, tempo energia e dinheiro. A relação entre as percepções do autor permite constatar que a coleção de processos e os fatores necessários para a condução da GC não é algo que possa ser promovido por todas as organizações.

Embora esta percepção possua certa lógica, devido a necessidade de investimentos nos diversos fatores supracitados, também é lógico que as organizações implementam práticas de GC ainda que não apresentem um programa formalizado voltado para o conhecimento. Teixeira Filho (2000) assinala o exemplo da TIM Celular Sul que criou um banco de talentos "mapeando as competências de todos os seus funcionários" (TEXEIRA FILHO, 2000, p. 75) e passou a utilizar esta base na alocação e substituição de pessoas em projetos, avaliação de desempenho e planos de treinamento. $O$ uso do banco de talentos, como exposto, se apresenta como uma prática que se alicerça sobre o conhecimento e não demanda a institucionalização de um programa de GC.

Portanto, parece factível inferir que o autor defende que o que não é para todos é a GC na íntegra, de forma institucionalizada e gerenciada, pois demanda investimentos generalizados em diversas frentes. No entanto, práticas de GC são plausíveis para todos. Isso por que em algum nível as empresas implementam práticas voltadas para o conhecimento. Os exemplos de Teixeira Filho (2000) quanto a TIM Celular Sul ou Xerox demonstram isso. Seja pela criação e uso do conhecimento advindo de banco de talentos - TIM Celular Sul - ou pela gestão eletrônica e documentos (GED) - XEROX -, tais práticas podem "ser para todos" sem que exista uma formalização da GC na organização.

Teixeira Filho (2000) também afirma que GC "não é tecnologia [...] criatividade e inovação [...] qualidade [...] marketing [...] documentação [...] gestão de recursos humanos" (TEIXEIRA FILHO, 2000, p. 12), mas está relacionada com todas. $O$ autor delimita decisivamente as fronteiras da GC para com outras áreas organizacionais, em específico a gestão de recursos

Perspectivas em Gestão \& Conhecimento, João Pessoa, v. 8, número especial, p. 167-182, out. 2018. 
humanos. Em contraponto, Davenport e Völpel (2001) afirmaram que "gerenciar conhecimento é gerenciar pessoas; gerenciar pessoas é gerenciar conhecimento" (DAVENPORT; VÖLPEL, 2001, p. 218, tradução nossa).

Essa segunda afirmativa permite compreender que a GC equivale a gestão de pessoas, o que pode acarretar em uma interpretação errônea, pois a GC se orienta ao conhecimento e a gestão de recursos humanos se volta para as pessoas, assim como posto na própria terminologia dessas áreas. Embora a distinção seja sutil, Teixeira Filho (2000) a demarca firmemente e permite refletir sobre as delimitações fronteiriças entre a GC e gestão de recursos humanos, assim como o faz em relação a inovação, remetendo a reflexão da obra de Nonaka e Takeuchi (1997) que tem o conhecimento como elemento propulsor da inovação, e não como equivalentes.

Quanto ao aspecto tecnológico, Teixeira Filho (2000) assinala que "A tecnologia sozinha é estéril. No seu uso inteligente é que está a diferença" (TEIXEIRA FILHO, 2000, p. 79). Embora a tecnologia seja referenciada pelo autor como elemento meio para a GC, seu modelo apresenta centralidade nessa dimensão de forma a utilizar o conhecimento para a constituição de uma memória organizacional e inteligência competitiva, o que corrobora com a perspectiva do autor de uso inteligente da tecnologia para a GC.

O modelo de Teixeira Filho (2000) se estabelece pelas dimensões conhecimento, tecnologia e organização (Figura 1). A dimensão conhecimento se subdivide em tácito, que reside na cabeça das pessoas, e explícito, registrado de alguma forma, corroborando com a perspectiva de Nonaka e Takeuchi (1997) e Davenport e Prusak (1998) quanto aos tipos de conhecimento.

Figura 1 - Modelo de Teixeira Filho (2000)

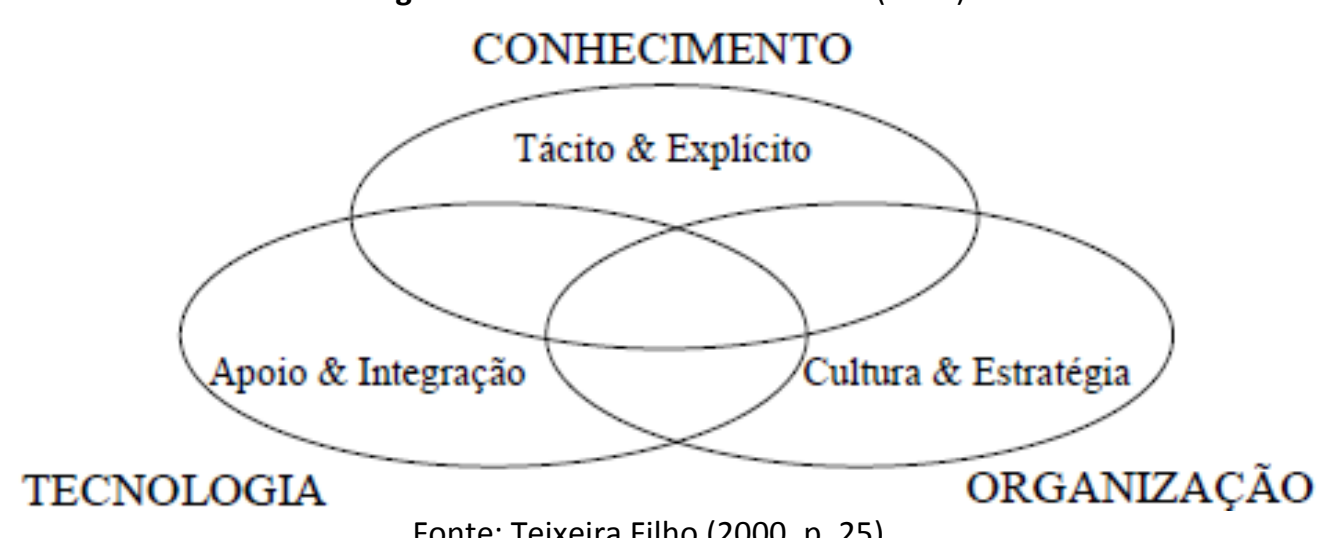

Fonte: Teixeira Filho (2000, p. 25)

A dimensão organização é constituída pelos fatores cultura e estratégia. Quanto a cultura, se tem que a "ponte da Gestão do Conhecimento para a administração de recursos humanos se dá, justamente, pela cultura organizacional" (TEIXEIRA FILHO, 2000, p. 47). Essa ótica é adere as dimensões Cultura e Gestão de recursos humanos da GC holística. Essa relação fora evidenciada de forma ampla por Anggia (2013) que apresenta a gestão de recursos humanos como um fator que influencia diversos aspectos organizacionais por atuar no cerne do conhecimento: as pessoas.

Em relação ao fator estratégia, para Teixeira Filho (2000) esse deve estabelecer os "temas de interesse para o processo" de GC (TEIXEIRA FILHO, 2000, p. 130), delimitando os conteúdos temáticos a serem gerenciados de forma com que esses estejam alinhados ao modelo de negócios e mercado de atuação da empresa. A visão da estratégia de Teixeira Filho (2000) se adere com duas dimensões da GC holística.

Perspectivas em Gestão \& Conhecimento, João Pessoa, v. 8, número especial, p. 167-182, out. 2018. 
Por um lado, se relaciona a dimensão Liderança e suporte da alta administração, pois a alta gerencia provê apontamentos quanto aos tipos de conhecimentos mais importantes para a empresa (DAVENPORT; DE LONG; BEERS, 1998), assinalando os campos de conhecimento a serem geridos (TERRA, 2005). Por outro lado se relaciona com a dimensão Estratégia, pois a estratégia de negócio deve estar alinhada à estratégia da GC (AKHAVAN; JAFARI; FATHIAN, 2006).

O fator tecnologia, como mencionado anteriormente, é o cerne do modelo de Teixeira Filho (2000). Sob esse fator o modelo apresenta 11 etapas - não é evidenciada a sequência de execução - para condução de um processo de implantação de GC orientado para a constituição de um sistema de informação (tecnologia). As etapas são: 1) "identificação de temas estratégicos"; 2) "mapeamento de fontes de informação gerais e específicas"; 3) "definição de indicadores para o processo de Gestão do Conhecimento"; 4) "modelagem do conteúdo"; 5) "identificação de gestores e responsáveis pelas informações"; 6) "definição do esquema de segurança e controle de acesso"; 7) "identificação de ciclos de atualização"; 8) "definição de séries históricas e informações agregadas de suporte à decisão"; 9) "definição dos componentes da infraestrutura tecnológica"; 10) "identificação de condicionantes e restrições"; e 11) "estruturação do glossário e indexação dos conteúdos" (TEIXEIRA FILHO, 2000, p. 131-133).

A "identificação de temas estratégicos" (1) (TEIXEIRA FILHO, 2000, p. 131) é aderente a dimensão Liderança e suporte da alta administração, pois prevê o apontamento quanto aos tipos de conhecimentos mais importantes para a empresa (DAVENPORT; DE LONG; BEERS, 1998), assinalando os campos de conhecimento a serem geridos (TERRA, 2005). Embora Davenport, De Long e Beers (1998) e Terra (2005) atribuam esta responsabilidade a alta gerência, Teixeira Filho (2000) não aponta a quem tais atribuições seriam destinadas.

O processo "mapeamento de fontes de informação gerais e específicas" (2) sucede a "identificação de temas estratégicos" (1) (TEIXEIRA FILHO, 2000, p. 131). A expectativa é de que o conteúdo a ser explorado pela GC quanto os temas estratégicos definidos sejam amparados por essas fontes, devendo as mesmas serem "seguras e de qualidade" (TEIXEIRA FILHO, 2000, p. 131). Pode-se inferir que o processo de mapeamento das fontes (2) depende da definição dos temas estratégicos (1), ocorrendo em sequência ao primeiro processo.

A "definição de indicadores para o processo de Gestão do Conhecimento" (3) (TEIXEIRA FILHO, 2000, p. 130) se refere a necessidade de estabelecer indicadores de performance para o projeto de GC, que na proposta do autor se trata um sistema de informação, e reportar os resultados a direção da organização. Visa avaliar o desempenho considerando a máxima de que "o que não se mede não tem como ser gerenciado" (TEIXEIRA FILHO, 2000, p. 131). Esse processo é aderente a dimensão Mensuração da GC holística, pois busca avaliar o desempenho organizacional (TERRA, 2005) e municiar a alta administração de informações que fomente seu apoio continuo (WONG, 2005).

O processo de "modelagem do conteúdo" (4) (TEIXEIRA FILHO, 2000, p. 130) resulta na definição das características dos conteúdos a serem geridos pela GC em relação aos temas estratégicos determinados (1); enquanto o processo de "identificação de gestores e responsáveis pelas informações" (5) (TEIXEIRA FILHO, 2000, p. 130) remete a necessidade de estabelecer responsáveis pelas informações a serem obtidas por meio das fontes de informação mapeadas (2).

O processo de "definição do esquema de segurança e controle de acesso" (6) (TEIXEIRA FILHO, 2000, p. 133) consiste na "segurança de informações [...] quanto a perdas e violações, intencionais ou não" (TEIXEIRA FILHO, 2000, p. 130), sendo aderente a dimensão Tecnologia da informação da GC holística, pois aborda o aspecto técnico da tecnologia pelo uso de banco de dados, intranet (SEDIGHI; ZAND, 2012) e internet (KUMAR; SINGH; HALEEM, 2015). No modelo de Teixeira Filho (2000) há ênfase na segurança de acesso ao sistema de informação.

Perspectivas em Gestão \& Conhecimento, João Pessoa, v. 8, número especial, p. 167-182, out. 2018. 
A "identificação de ciclos de atualização" (7) (TEIXEIRA FILHO, 2000, p. 133) corresponde a especificação de periodicidade de atualização das fontes de informação (processo 2) e conteúdo (processo 4) (TEIXEIRA FILHO, 2000). A “definição de séries históricas e informações agregadas de suporte à decisão" (8) (TEIXEIRA FILHO, 2000, p. 133) resulta na perspectiva histórica das informações armazenadas no sistema, devendo essas serem definidas para suporte na tomada de decisão (TEIXEIRA FILHO, 2000).

O processo de "definição dos componentes da infraestrutura tecnológica" (9) (TEIXEIRA FILHO, 2000, p. 133) e a "identificação de condicionantes e restrições" (10) (TEIXEIRA FILHO, 2000, p. 133) correspondem a questões arquiteturais tecnológicas - tipo de banco de dados, servidores, outros - que permitem estimativas relacionadas a custos e plataforma tecnológica (9) e restrições orçamentárias e políticas (10), dentre outros fatores de gestão de projetos que circundam o desenvolvimento de um sistema de informação (TEIXEIRA FILHO, 2000).

Por fim, o processo "estruturação do glossário e indexação dos conteúdos" (7) (TEIXEIRA FILHO, 2000, p. 133) remete a determinação das formas de busca no sistema de informação para recuperação do conteúdo armazenado (TEIXEIRA FILHO, 2000). Remete a dimensão Tecnologia da informação da GC holística, pois aborda a necessidade de organização do conhecimento explícito (KUMAR; SINGH; HALEEM, 2015) de forma que esses possam ser recuperadas por meio do uso de palavras-chave, dicionários de sinônimos e tesauros fazendo uso de outros artifícios de indexação como a taxonomia (DAVENPORT; DE LONG; BEERS, 1998; ARIF; SHALHOUB, 2014).

Para Teixeira Filho (2000) um projeto de sistema de informação de GC deve ter um diretor do conhecimento (Chief Knowledge Officer - CKO) como responsável e analistas de informação para gerir as fontes e informações a serem obtidas nas mesmas. Segundo o autor cada "parte do conteúdo mapeado e cada parte do processo de Gestão do Conhecimento tem que ter seu responsável devidamente identificado, com atribuições claramente definidas" (TEIXEIRA FILHO, 2000, p. 132).

A determinação desses perfis é aderente a dimensão Equipe de gestão do conhecimento da GC holística, pois visa o estabelecimento de uma equipe de profissionais voltados para a promoção do conhecimento organizacional (DAVENPORT; DE LONG; BEERS, 1998) com papéis específicos (WONG; ASPINWALL, 2005; VALMOHAMMADI, 2010) e responsabilidades (ARIF; SHALHOUB, 2014).

Ademais, Teixeira Filho (2000) salienta a relevância de promover o desenvolvimento e implementação desse sistema de informação considerando um projeto piloto que se adeque às particularidades organizacionais, sendo aderente a dimensão Projeto piloto da GC holística. Antes de promover uma implantação da GC em toda a organização um projeto piloto pode ser considerado, visando identificar melhores práticas de forma a aumentar o potencial de acerto por meio de lições aprendidas (SKYRME; AMIDON, 1997; AKHAVAN; JAFARI; FATHIAN, 2006).

\subsection{Resultados}

O modelo de Teixeira Filho (2000) evidencia processos essencialmente voltados para a constituição de um sistema de informação para a GC e descreve aspectos a serem considerados nesse desenvolvimento. Por um lado, embora o modelo do autor evidencie a presença do conhecimento tácito e explícito, a proposta se sedimenta na constituição de uma memória organizacional tecnológica e, portanto, o modelo converge para o tratamento do conhecimento especificamente explícito.

Por outro lado, a totalidade da obra descreve diversos aspectos da GC e, ainda que a perspectiva tecnológica seja o pilar do modelo, o modelo apresenta outras dimensões como estratégia e cultura. Por síntese, o modelo apresenta as seguintes dimensões da GC holística.

Perspectivas em Gestão \& Conhecimento, João Pessoa, v. 8, número especial, p. 167-182, out. 2018. 
- Estratégia: sim. O conteúdo (conhecimento) a ser gerido no sistema de informação para a GC deve estar alinhado ao modelo de negócios e mercado de atuação da empresa;

- Liderança e suporte da alta administração: sim. Prevê o estabelecimento dos "temas de interesse para o processo" - conteúdo do conhecimento - de GC (TEIXEIRA FILHO, 2000, p. 130) como sendo uma orientação estratégica que apresenta relação com a alta gerência;

- Equipe de gestão do conhecimento: sim. Estabelecida pelos perfis de diretor do conhecimento e analistas de informação;

- Recursos (financeiro, humano, material e tempo): não identificada no modelo;

- Processos e atividades: não. Embora seja delimitada a criação, disseminação e utilização do conhecimento, conforme conceito de GC proposto pelo autor, esses não são sustentados no modelo;

- Gestão de recursos humanos: não identificada no modelo;

- Treinamento e educação: não identificada no modelo;

- Motivação: não identificada no modelo;

- Trabalho em equipe: não identificada no modelo;

- Cultura: sim. Estabelecida pela perspectiva de que a "ponte da Gestão do Conhecimento para a administração de recursos humanos se dá, justamente, pela cultura organizacional" (TEIXEIRA FILHO, 2000, p. 47);

- Tecnologia da informação: sim. Presente na totalidade da obra e central do modelo proposto;

- Mensuração: sim. Necessidade de definir indicadores de performance para o projeto tecnológico de GC;

- Projeto Piloto: sim. Recomendado como sendo útil para adequar o projeto às particularidades organizacionais.

Mediante a análise promovida sobre o modelo de Teixeira Filho (2000) os fatores acima ponderados são dispostos no Gráfico 1, que exprime a adesão do referido modelo as dimensões da GC holística atinentes a essa pesquisa. A presença de uma dimensão a posiciona na escala 1 (extremidade externa) do gráfico e sua ausência a assinala na escala 0 (centro).

Gráfico 1 - Adesão do modelo de Teixeira Filho (2000) as dimensões da gestão do conhecimento

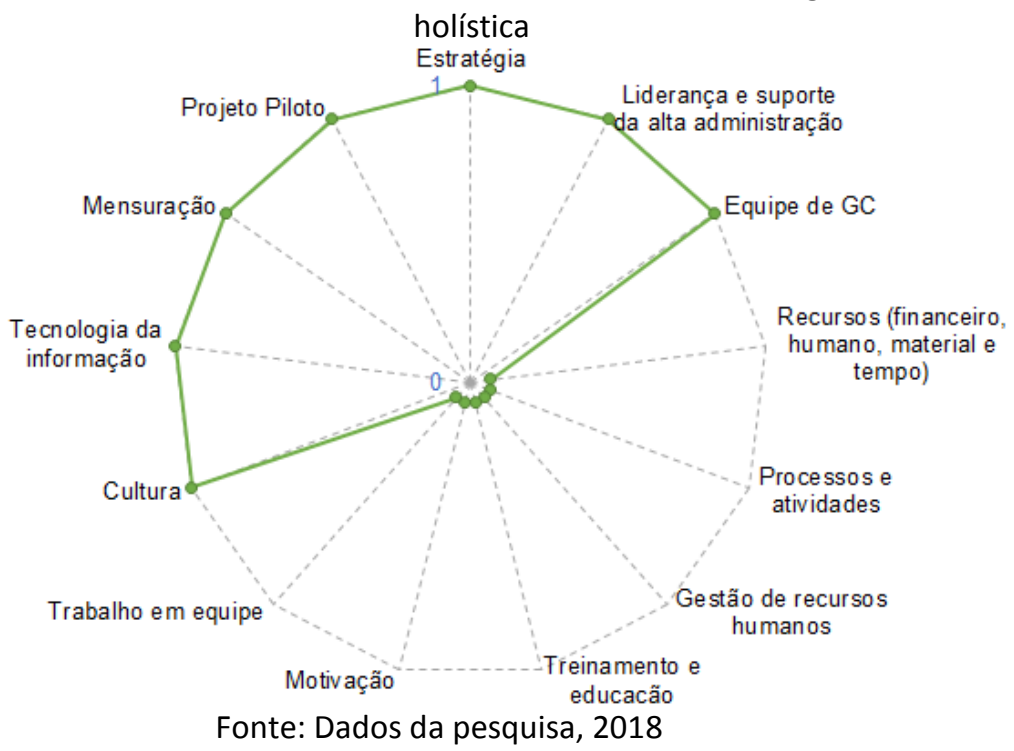

Perspectivas em Gestão \& Conhecimento, João Pessoa, v. 8, número especial, p. 167-182, out. 2018. 
Assim, o modelo de Teixeira Filho (2000) contempla as dimensões Estratégia, Liderança e suporte da alta administração, Equipe de GC, Cultura, Tecnologia da informação, Mensuração e Projeto Piloto, o remetendo ao percentual de 53,8\% (7 do total de 13 dimensões) de adesão ao que considera por GC holística, conforme caracterização apresentada nessa pesquisa.

\section{CONSIDERAÇÕES FINAIS}

Esse estudo se propôs a analisar a aderência do modelo de Teixeira Filho (2000) aos fatores da GC holística. Esse exame, promovido por meio da Análise de Conteúdo de cunho qualitativo, percorreu integralmente a referida obra identificando os indícios que aderem o modelo as dimensões analisadas. Após essa trajetória, os fatores foram contabilizados e expostos em uma perspectiva gráfica, se valendo da quantificação. Visando maior rigor metodológico, essa pesquisa fez uso da natureza descritiva, primando pela descrição dos fatos relativos ao exame da obra.

Primeiramente, se esclarece que não há intento em promover crédito ou imputar a ausência desse na obra estudada. A ideia não é analisar a relevância da obra, pois apresenta reconhecimento por suas citações em estudos relacionados a GC. O que se almeja é avaliar o quão essa se adere ao que se denomina por holísmo na GC.

Por resultado, a obra de Teixeira Filho (2000) adere a 53,8\% das dimensões da visão holística, o que remete há necessidade de desenvolvimento de modelos mais abrangentes, orientados a todas as dimensões da GC e suas relações de forma a atingir o hólus dessa temática no âmbito organizacional.

Acredita-se que essa pesquisa venha a contribuir por apontar um delineamento da GC a visão holística de forma a permitir um avanço da compreensão referente ao ajustamento dessas duas correntes teóricas. Também por analisar as dimensões da GC holística e a aderência de uma das principais obras nacionais relacionadas ao conhecimento. Todavia, a análise de outros modelos seguindo os procedimentos aqui adotados, bem como o desenvolvimento de um modelo holístico, são propostas de pesquisas futuras orientadas ao intento de convergir a GC ao hólus das organizações do conhecimento.

\section{AGRADECIMENTOS}

À FAPEMIG, à CAPES, ao CNPq e à FUMEC.

\section{REFERÊNCIAS}

ABBASZADEH, M. A.; EBRAHIMI, M.; FOTOUHI, H. Developing a causal model of critical success factors for knowledge management implementation. In: International Conference on Education and Management Technology ICEMT 2010, Cairo. Anais... Cairo, 2010. p. 701-705.

AKHAVAN, P.; JAFARI, M.; FATHIAN, M. Critical success factors of knowledge management systems: A multi-case analysis. European Business Review, v.18, n. 2, p. 97-113, 2006.

AL-MABROUK, K. Critical success factors affecting knowledge management adoption: A review of the literature. In Innovations in Information Technology IITFALSO, Dubai. Anais... Dubai, 2006.

\footnotetext{
Perspectivas em Gestão \& Conhecimento, João Pessoa, v. 8, número especial, p. 167-182, out. 2018.
} 
ALTAHER, A. M. Critical success factors of implementation knowledge management process. In: International Conference on Information Society, i-Society 2010, Londres. Anais... Londres, 2010. p. 340-348.

ALVES, F. A. B. Proposta de melhoria na gestão do planejamento do processo de compras em uma instituição de ensino. 2017, 128 f. Dissertação (Mestrado em Sistemas de Informação) Programa de Pós-graduação em Sistemas de Informação, Universidade Federal Fluminense, 2017.

ANDRADE, R.; AMBONI, N. TGA - Teoria Geral da Administração. Elsevier Brasil, 2017 [livro eletrônico].

ANGELONI, M. T. (org.). Organizações do conhecimento: infra-estrutura, pessoas e tecnologia. São Paulo: Saraiva, 2002.

ANGGIA, P. et al. Identifying critical success factors for knowledge management implementation in organization: A survey paper. In: International Conference on Advanced Computer Science and Information Systems ICACSIS 2013, Bali. Anais... Bali, 2013. p. 83-88.

ARIF, M. J.; SHALHOUB, M. H. B. Critical success factors with its effective role in knowledge management initiatives in public and private organizations in saudi Arabia: experts perspectives. Life Science Journal, v.11, n. 6, p. 636-645, 2014.

BARDIN, L. Análise de conteúdo. Lisboa: Edições 70, 1977.

BARROS, A. J. S.; LEHFELD, N. A. S. Fundamentos de metodologia científica. 3. ed. São Paulo, 2007.

BEHRENS, M. A. A prática pedagógica e o desafio do paradigma emergente. Revista Brasileira de Estudos Pedagógicos, v. 80, n. 196, p. 383-403, 2007.

CAJUEIRO, J. L. G. Modelo de gestão do conhecimento para instituições de ensino superior. 2008. 152f. Tese (Doutorado em Engenharia de Produção) -. Programa de Pós-graduação em Engenharia de Produção, Universidade Federal de Pernambuco, 2008.

CAPRA, F. A teia da vida: uma nova compreensão científica dos sistemas vivos. 9 ed. São Paulo: Cultrix, 2000.

COIMBRA, J. Á. A. Considerações sobre a Interdisciplinaridade. USP: Interdisciplinaridade em Ciências Ambiental, p. 52-70,1996.

CORMEN, T. H. Algoritmos: teoria e pratica. 1. ed. Rio de Janeiro: Campus, 2002.

COSTA, P. E. C. Desenvolvimento de um modelo de gestão do conhecimento para a melhoria do processo de desenvolvimento de produtos, 2005, $107 \mathrm{f}$. Dissertação (Mestrado em Engenharia de Produção) -. Programa de Pós-graduação em Engenharia de Produção, Universidade Federal do Rio Grande do Norte, 2005.

Perspectivas em Gestão \& Conhecimento, João Pessoa, v. 8, número especial, p. 167-182, out. 2018. 
CREMA, R. Abordagem holística: integração do método analítico e sintético. In: BRANDÃO, D.; CREMA, R. (orgs). O novo paradigma holístico: ciência, filosofia, arte e mística. São Paulo: Summus, p. 83-99, 1991.

CREMA, R. Introdução à visão holística: breve relato de viagem do velho ao novo paradigma. 6 ed. São Paulo: Summus, 2015 [livro eletrônico].

CRESWELL, J. W. Projeto de pesquisa: métodos qualitativo, quantitativo e misto. 2. ed. Porto Alegre: Artmed, 2007.

DAVENPORT, T. H.; DE LONG, D. W.; BEERS, M. C. Successfull knowledge management projects. Sloan Management Review, v.39, n. 2, p. 43-57, 1998.

DAVENPORT, T. H.; VÖLPEL, S. C. The rise of knowledge towards attention management. Journal of Knowledge Management, v. 5, n. 3, p. 212-221, 2001.

DAVENPORT, T. H; PRUSAK, L. Conhecimento empresarial: como as organizações gerenciam o seu capital intelectual. 11. ed. Rio de Janeiro: Campus, 1998.

FERREIRA, V. C. P. et al. Modelos de gestão. 3 ed. Rio de Janeiro: Editora FGV, 2009.

FLACH, C. R. C.; BEHRENS, M. A. Paradigmas educacionais e sua influência na prática pedagógica. In: Congresso Ibero-Americano Sobre Violências Na Escola, 3, 2008, Curitiba. Anais... Curitiba, 2008.

FLICK, U. Uma introdução à pesquisa qualitativa. 2. ed. Porto Alegre: Bookman, 2004.

GAI, S.; XU, C. Research of critical success factors for implementing knowledge management in China. In: International Conference on Information Management, Innovation Management and Industrial Engineering, ICIII 2009, Xi'an. Anais... Xi'an, 2009. p. 561-564.

GASKELL, G.; BAUER, M. W. Pesquisa qualitativa com texto, imagem e som. Petrópolis: Vozes, p. 137-155, 2002.

GIL, A. C. Métodos e técnicas de pesquisa social. 4. ed. São Paulo: Atlas, 2002.

HANASHIRO, A. Proposta de modelo de gestão do conhecimento no piso de fábrica: estudo de caso de Kaizen em empresa do setor automotivo. 2005, 137 f. Dissertação (Mestrado em Engenharia Mecânica) - Programa de Pós-graduação em Engenharia Mecânica, Universidade Federal do Paraná, 2005.

HEISIG, P. Harmonisation of knowledge management - comparing $160 \mathrm{KM}$ frameworks around the globe. Journal of knowledge management, v. 13, n. 4, p. 4-31, 2009.

HOLSAPPLE, C. W.; JOSHI, K. D. Description and analysis of existing knowledge management frameworks. In: Systems Sciences, 1999. In: 32nd Annual Hawaii International Conference HICSS-32, Maui. Anais... Maui, 1999. p. 15.

KERLINGER, F. N. Metodologia da pesquisa em ciências sociais: um tratamento conceitual. Editora Pedagógica e Universitária Ltda, 1979.

Perspectivas em Gestão \& Conhecimento, João Pessoa, v. 8, número especial, p. 167-182, out. 2018. 
KUMAR, S.; SINGH, V.; HALEEM, A. Critical success factors of knowledge management: modelling and comparison using various techniques. International Journal of Industrial and Systems Engineering, v.21, n. 2, p. 180-206, 2015.

LIN, Y-C.; LIN, L-K. Critical success factors for knowledge management studies in construction. 2006. In: 23rd International Symposium on Robotics and Automation in Construction ISARC 2006, Tokyo, Anais... Tokyo, 2006. p. 768-772.

MACIEL, C.; SILVA, A. Gerenciando pessoas utilizando modelos holísticos. Revista da Administração Contemporânea, v. 12, n. 1, p. 35-58, 2008.

MARCONI, M. A.; LAKATOS, E. M. Fundamentos de metodologia científica. 5. ed. São Paulo: Atlas, 2003.

MELO, A. M.; MEDEIROS, M. V. B. Um modelo de uso de ferramentas de Inteligência Competitiva. Revista de Administração e Contabilidade, v. 4, n. 7, p. 112-135, 2017.

MINAYO, M. C. S. O desafio do conhecimento: pesquisa qualitativa em saúde. 5. ed. São Paulo: Hucitec, 1998.

NAGHAVI, M.; DASTAVIZ, A. H.; NEZAKATI, H. Relationships among critical success factors of knowledge management and organizational performance. Journal of Applied Sciences, v.13, n. 5, p. 755-759, 2013.

NONAKA, I.; TAKEUCHI, H. Criação de conhecimento na empresa: como as empresas japonesas geram a dinâmica da inovação. 10 ed. Rio de Janeiro: Editora Campus, 1997.

OLIVEIRA, P. H. et al. Gestão do conhecimento orientada para a estratégia de inovação de produtos tecnológicos: o caso da Invent Vision. Revista de Administração e Inovação, v. 9, n. 4, p. 153-176, 2012.

PEREIRA, M. F. A gestão organizacional em busca do comportamento holístico. In: ANGELONI, M. T. (Org.). Organizações do conhecimento: infra-estrutura, pessoas e tecnologia. São Paulo: Saraiva, p. 2-28, 2002.

RUBENSTEIN-MONTANO, B. et al. A systems thinking framework for knowledge management. Decision support systems, v. 31, n. 1, p. 5-16, 2001.

SEDIGHI, M.; ZAND, F. Knowledge management: Review of the Critical Success Factors and development of a conceptual classification model. In: International Conference on ICT and Knowledge Engineering, Bangkok. Anais... Bangkok, 2012. p. 1-9.

SILVA, R. O. Teorias da administração. 2 ed. São Paulo: Pearson Education do Brasil, 2013.

SKYRME, D.; AMIDON, D. The knowledge agenda. Journal of Knowledge Management, v.1, n. 1, p. 27-37, 1997.

Perspectivas em Gestão \& Conhecimento, João Pessoa, v. 8, número especial, p. 167-182, out. 2018. 
TEIXEIRA FILHO, J. Gerenciando conhecimento: como a empresa pode usar a memória organizacional e a inteligência competitiva no desenvolvimento de negócios. 2. ed. Rio de Janeiro: SENAC, 2000.

TERRA, J. C. C. Gestão do conhecimento: o grande desafio empresarial. 5ed. Rio de Janeiro: Elsevier, 2005.

VALMOHAMMADI C. Investigation and assessment of critical success factors of knowledge management implementation in Iranian small-to-medium sized enterprises. Journal of Applied Sciences, v.10, n. 19, p. 2290-2296, 2010.

VERGARA, S. C. Sobre a intuição na tomada de decisão. Cadernos EBAP, n. 62, 1993.

WAI, Y. M.; HONG, A. N. H.; DIN, S. B. Critical success factors and perceived benefits of knowledge management implementation: Towards a conceptual framework. Australian Journal of Basic and Applied Sciences, v.5, n. 10, p. 754-760, 2011.

WEIL, P. O novo paradigma holístico: ondas a procura do mar. In: BRANDÃO, D.; CREMA, R. (orgs). O novo paradigma holístico: ciência, filosofia, arte e mística. São Paulo: Summus, p. 1438, 1991.

WONG, K. Y. Critical success factors for implementing knowledge management in small and medium enterprises. Industrial Management \& Data Systems, v.105, n. 3, p. 261-279, 2005.

WONG, K. Y; ASPINWALL, E. An empirical study of the important factors for knowledgemanagement adoption in the SME Sector. Journal of Knowledge Management, v.9, n. 3, p. 6482, 2005.

ZIEBA, M.; ZIEBA, K. Knowledge management critical success factors and the innovativeness of KIBS companies. Engineering Economics, v.25, n. 4, p. 458-465, 2014.

ZIVIANI, N. Projeto de algoritmos com implementações em pascal e c. 3. ed., Cengage Learning, 2014.

Artigo recebido em 09/10/2018 e aceito para publicação em 29/10/2018 